\title{
Model-independent search for neutrino sources with the ANTARES neutrino telescope
}

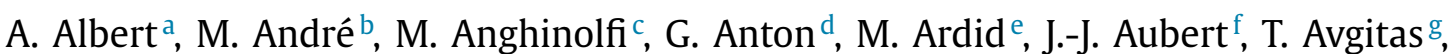
B. Baret ${ }^{g}$, J. Barrios-Martí ${ }^{h}$, S. Basa ${ }^{i}$, V. Bertinn ${ }^{f}$, S. Biagi ${ }^{j}$, R. Bormuth ${ }^{\mathrm{k}, \mathrm{l}}$, S. Bourret ${ }^{\mathrm{g}}$, M.C. Bouwhuis ${ }^{k}$, R. Bruijn ${ }^{\mathrm{k}, \mathrm{m}}$, J. Brunner ${ }^{\mathrm{f}}$, J. Busto ${ }^{\mathrm{f}}$, A. Capone ${ }^{\mathrm{n}, \mathrm{o}}$, L. Caramete ${ }^{\mathrm{p}}$, J. Carr $^{\mathrm{f}}$, S. Celli ${ }^{\mathrm{n}, \mathrm{o}, \mathrm{q}}$, T. Chiarusi ${ }^{\mathrm{r}}$, M. Circella ${ }^{\mathrm{s}}$, J.A.B. Coelhog ${ }^{g}$, A. Coleiro ${ }^{g}$, R. Coniglione ${ }^{\mathrm{j}}$, H. Costantini ${ }^{f}$, P. Coyle ${ }^{f}$, A. Creusot ${ }^{g}$, A. Deschamps ${ }^{t}$, G. De Bonis ${ }^{n, o}$, C. Distefano ${ }^{j}$, I. Di Palma $^{\mathrm{n}, \mathrm{o}}$, C. Donzaud ${ }^{\mathrm{g}, \mathrm{u}}$, D. Dornic ${ }^{\mathrm{f}}$, D. Drouhin ${ }^{\mathrm{a}}$, T. Eberl ${ }^{\mathrm{d}, *}$, I. El Bojaddaini ${ }^{\mathrm{v}}$, D. Elsässer ${ }^{\mathrm{w}}$, A. Enzenhöfer ${ }^{\mathrm{f}}$, I. Felis ${ }^{\mathrm{e}}$, L.A. Fusco ${ }^{\mathrm{r}, \mathrm{x}}, \mathrm{S}$. Galatà ${ }^{\mathrm{g}}$, P. Gay ${ }^{\mathrm{y}, \mathrm{g}}, \mathrm{S}$. Geißelsöder ${ }^{\mathrm{d}, *}$, K. Geyer $^{\mathrm{d}}$, V. Giordano ${ }^{z}$, A. Gleixner ${ }^{\mathrm{d}}$, H. Glotin ${ }^{\mathrm{A}, \mathrm{C}, \mathrm{B}}$, T. Grégoires, R. Gracia Ruiz" ${ }^{\mathrm{g}}$, K. Graf ${ }^{\mathrm{d}}$, S. Hallmann ${ }^{\mathrm{d}}$, H. van Haren ${ }^{\mathrm{D}}$, A.J. Heijboer ${ }^{\mathrm{k}}$, Y. Hello ${ }^{\mathrm{t}}$, J.J. Hernández-Rey ${ }^{\mathrm{h}}$, J. Hößl ${ }^{\mathrm{d}}$, J. Hofestädt ${ }^{\mathrm{d}}$, C. Hugon ${ }^{\mathrm{c}, \mathrm{E}}$, G. Illuminati ${ }^{\mathrm{n}, \mathrm{o}, \mathrm{h}}$, C.W. James ${ }^{\mathrm{d}}$, M. de Jong ${ }^{\mathrm{k}, \mathrm{l}}$, M. Jongen ${ }^{\mathrm{k}}$, M. Kadler ${ }^{\text {w }}$, O. Kalekin ${ }^{\mathrm{d}}$, U. Katz ${ }^{\mathrm{d}}$, D. Kießling ${ }^{\mathrm{d}}$, A. Kouchner ${ }^{\mathrm{g}, \mathrm{C}}$, M. Kreter ${ }^{\mathrm{w}}$, I. Kreykenbohm ${ }^{\mathrm{F}}$, V. Kulikovskiy ${ }^{\mathrm{j}, \mathrm{G}}$, C. Lachaud $^{\mathrm{g}}$, R. Lahmann ${ }^{\mathrm{d}}$, D. Lefèvre ${ }^{\mathrm{H}, \mathrm{I}}$, E. Leonora ${ }^{\mathrm{Z} J}$, M. Lotze ${ }^{\mathrm{h}}, \mathrm{S}$. Loucatos ${ }^{\mathrm{K}, \mathrm{g}}$, M. Marcelin ${ }^{\mathrm{i}}$, A. Margiotta ${ }^{\mathrm{r}, \mathrm{X}}$, A. Marinelli ${ }^{\mathrm{L}, \mathrm{M}}$, J.A. Martínez-Mora ${ }^{\mathrm{e}}$,

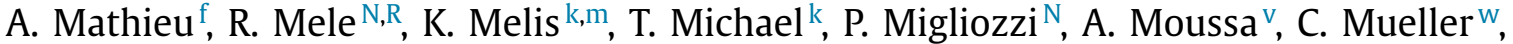

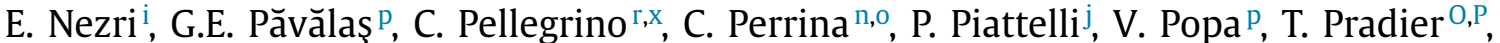
L. Quinnn ${ }^{f}$, C. Racca ${ }^{a}$, G. Riccobene ${ }^{j}$, K. Roensch ${ }^{\mathrm{d}}$, A. Sánchez-Losa ${ }^{\mathrm{s}}$, M. Saldaña ${ }^{\mathrm{e}}$, I. Salvadori ${ }^{f}$, D.F.E. Samtleben ${ }^{\mathrm{k}, \mathrm{l}}$, M. Sanguineti ${ }^{\mathrm{c}, \mathrm{E}}$, P. Sapienza ${ }^{\mathrm{j}}$, J. Schnabel ${ }^{\mathrm{d}}$, F. Schüssler ${ }^{\mathrm{K}}$, T. Seitz ${ }^{d}$, C. Sieger ${ }^{d}$, M. Spurio ${ }^{\mathrm{r}, \mathrm{x}}$, Th. Stolarczyk ${ }^{\mathrm{K}}$, M. Taiuti ${ }^{\mathrm{c}, \mathrm{E}}$, , Y. Tayalati $^{\mathrm{Q}}$, A. Trovato $^{\mathrm{j}}$, M. Tselengidou ${ }^{\mathrm{d}}$, D. Turpin ${ }^{\mathrm{f}}$, C. Tönnis ${ }^{\mathrm{h}}$, B. Vallage ${ }^{\mathrm{K}, \mathrm{g}}$, , C. Vallée $^{\mathrm{f}}$, V. Van Elewyck ${ }^{\mathrm{g}, \mathrm{C}}$, D. Vivolo $^{\mathrm{N}, \mathrm{R}}$, A. Vizzoca ${ }^{\mathrm{n}, \mathrm{o}}$, S. Wagner ${ }^{\mathrm{d}}$, J. Wilms ${ }^{\mathrm{F}}$, J.D. Zornoza ${ }^{\mathrm{h}}$, J. Zúñiga ${ }^{\mathrm{h}}$

\footnotetext{
${ }^{a}$ GRPHE - Université de Haute Alsace - Institut universitaire de technologie de Colmar, 34 rue du Grillenbreit BP Colmar 50568 - 68008, France

${ }^{\mathrm{b}}$ Technical University of Catalonia, Laboratory of Applied Bioacoustics, Rambla Exposició,08800 Vilanova i la Geltrú, Barcelona, Spain

' INFN - Sezione di Genova, Via Dodecaneso 33, Genova 16146, Italy

${ }^{\mathrm{d}}$ Friedrich-Alexander-Universität Erlangen-Nürnberg, Erlangen Centre for Astroparticle Physics, Erwin-Rommel-Str. 1, Erlangen 91058, Germany

e Institut d'Investigació per a la Gestió Integrada de les Zones Costaneres (IGIC) - Universitat Politècnica de València. C/ Paranimf 1, Gandia 46730, Spain ${ }^{\mathrm{f}}$ Aix-Marseille Université, CNRS/IN2P3, CPPM UMR 7346, Marseille 13288, France

${ }^{g}$ APC, Université Paris Diderot, CNRS/IN2P3, CEA/IRFU, Observatoire de Paris, Sorbonne Paris Cité, Paris 75205, France

${ }^{\mathrm{h}}$ IFIC - Instituto de Física Corpuscular (CSIC - Universitat de València) c/ Catedrático José Beltrán, 2 E-46980 Paterna, Valencia, Spain

${ }^{\mathrm{i}}$ LAM - Laboratoire d'Astrophysique de Marseille, Pôle de l'Étoile Site de Château-Gombert, rue Frédéric Joliot-Curie 38, Marseille Cedex 13 13388, France

j INFN - Laboratori Nazionali del Sud (LNS), Via S. Sofia 62, Catania 95123, Italy

${ }^{\mathrm{k}}$ Nikhef, Science Park, Amsterdam, The Netherlands

${ }^{1}$ Huygens-Kamerlingh Onnes Laboratorium, Universiteit Leiden, The Netherlands

m Universiteit van Amsterdam, Instituut voor Hoge-Energie Fysica, Science Park 105, XG Amsterdam 1098, The Netherlands

${ }^{\mathrm{n}}$ INFN -Sezione di Roma, P.le Aldo Moro 2, Roma 00185, Italy

${ }^{\circ}$ Dipartimento di Fisica dell'Università La Sapienza, P.le Aldo Moro 2, Roma 00185, Italy

P Institute for Space Science, RO-077125 Bucharest, Măgurele, Romania

${ }^{\mathrm{q}}$ Gran Sasso Science Institute, Viale Francesco Crispi 7, L’Aquila 00167, Italy

${ }^{\mathrm{r}}$ INFN - Sezione di Bologna, Viale Berti-Pichat 6/2, Bologna 40127, Italy

${ }^{\mathrm{s}}$ INFN - Sezione di Bari, Via E. Orabona 4, Bari 70126, Italy

${ }^{\mathrm{t}}$ Géoazur, UCA, CNRS, IRD, Observatoire de la Côte d'Azur, Sophia Antipolis, France
}

\footnotetext{
* Corresponding author.

E-mail addresses: stefan.geisselsoeder@fau.de (T. Eberl),stefan.geisselsoeder@ fau.de (S. Geißelsöder).
} 
"Univ. Paris-Sud, 91405 Orsay Cedex, France

${ }^{v}$ University Mohammed I, Laboratory of Physics of Matter and Radiations, B.P.717, Oujda 6000, Morocco

${ }^{w}$ Institut für Theoretische Physik und Astrophysik, Universität Würzburg, Emil-Fischer Str. 31, Würzburg 97074, Germany

x Dipartimento di Fisica e Astronomia dell'Università, Viale Berti Pichat 6/2, Bologna 40127, Italy

${ }^{y}$ Laboratoire de Physique Corpusculaire, Clermont Univertsité, Université Blaise Pascal, CNRS/IN2P3, BP 10448, Clermont-Ferrand F-63000, France

${ }^{\mathrm{z}}$ INFN - Sezione di Catania, Viale Andrea Doria 6, Catania 95125, Italy

${ }^{\mathrm{A}}$ LSIS, Aix Marseille Université CNRS ENSAM LSIS UMR 7296 Marseille 13397, France

${ }^{\mathrm{B}}$ Université de Toulon CNRS LSIS UMR La Garde 7296 83957, France

${ }^{C}$ Institut Universitaire de France, Paris 75005, France

${ }^{D}$ Royal Netherlands Institute for Sea Research (NIOZ), Landsdiep 4, 1797 SZ 't Horntje (Texel), The Netherlands

E Dipartimento di Fisica dell'Università, Via Dodecaneso 33, Genova 16146, Italy

${ }^{\mathrm{F}}$ Dr. Remeis-Sternwarte and ECAP, Universität Erlangen-Nürnberg, Sternwartstr. 7, Bamberg 96049, Germany

${ }^{\mathrm{G}}$ Moscow State University, Skobeltsyn Institute of Nuclear Physics, Leninskie gory, Moscow 119991, Russia

${ }^{\mathrm{H}}$ Mediterranean Institute of Oceanography (MIO), Aix-Marseille University, 13288, Marseille, Cedex 9, France

${ }^{1}$ Université du Sud Toulon-Var, 83957, La Garde Cedex, France CNRS-INSU/IRD UM 110, France

J Dipartimento di Fisica ed Astronomia dell'Università, Viale Andrea Doria 6, Catania 95125, Italy

${ }^{\mathrm{K}}$ Direction des Sciences de la Matière - Institut de recherche sur les lois fondamentales de l'Univers - Service de Physique des Particules, CEA Saclay,

Gif-sur-Yvette Cedex 91191, France

L INFN - Sezione di Pisa, Largo B. Pontecorvo 3, Pisa 56127, Italy

${ }^{\mathrm{M}}$ Dipartimento di Fisica dell'Università, Largo B. Pontecorvo 3, Pisa 56127, Italy

${ }^{\mathrm{N}}$ INFN -Sezione di Napoli, Via Cintia Napoli 80126, Italy

${ }^{0}$ Université de Strasbourg, IPHC, 23 rue du Loess 67037 Strasbourg, France

${ }^{\mathrm{P}}$ CNRS, UMR7178, Strasbourg 67037, France

${ }^{Q}$ University Mohammed V in Rabat, Faculty of Sciences, 4 av. Ibn Battouta, B.P. 1014, Rabat R.P. 10000, Morocco

${ }^{\mathrm{R}}$ Dipartimento di Fisica dell'Università Federico II di Napoli, Via Cintia 80126, Napoli, Italy

\section{A R T I C L E I N F O}

\section{Article history:}

Received 27 February 2017

Revised 14 April 2019

Accepted 12 June 2019

Available online 13 June 2019

\section{MSC:}

85-04

$85-05$

85-08

Keywords:

Neutrino astronomy

Astroparticle physics

Pattern recognition

Anisotropy

\begin{abstract}
A B S T R A C T
A novel method to analyse the spatial distribution of neutrino candidates recorded with the ANTARES neutrino telescope is introduced, searching for an excess of neutrinos in a region of arbitrary size and shape from any direction in the sky. Techniques originating from the domains of machine learning, pattern recognition and image processing are used to purify the sample of neutrino candidates and for the analysis of the obtained skymap. In contrast to a dedicated search for a specific neutrino emission model, this approach is sensitive to a wide range of possible morphologies of potential sources of high-energy neutrino emission. The application of these methods to ANTARES data yields a large-scale excess with a post-trial significance of $2.5 \sigma$. Applied to public data from IceCube in its IC40 configuration, an excess consistent with the results from ANTARES is observed with a post-trial significance of $2.1 \sigma$.
\end{abstract}

(c) 2019 Elsevier B.V. All rights reserved.

\section{Introduction}

Since the recent discovery of a diffuse high-energy astrophysical neutrino flux by the IceCube Collaboration [1-3], neutrino astronomy has established itself as a new discipline. Due to the statistical limitations implied by a new observational tool that has just overcome its initial detection threshold, the spectral and spatial properties of the discovered flux are still not well constrained.

The high-energy starting event (HESE) analysis [2], which is most sensitive to the Southern sky but has a poor spatial resolution, measures a best-fit diffuse signal of $d N_{\nu} / d E \propto E^{\Gamma}$ with $\Gamma=$ -2.5 [4]. The flux observed from the Northern Sky with a higher energy threshold of about $200 \mathrm{TeV}$ in the muon channel exhibits a harder spectral index of about $\Gamma=-2.13 \pm 0.13$ [5].

A first analysis has shown that the spatial distribution of HESE events is consistent with an isotropic distribution [6].

While there seems to be some evidence for an excess at low galactic latitudes [7], favouring a galactic contribution with a softer spectrum and an extragalactic contribution with a harder spectrum [8], the origin of the various contributions remains open.

Several analyses with the goal to reveal the origin of the astrophysical neutrinos have been performed. Time-integrated searches for point-like and extended bright sources by ANTARES [9], IceCube [10] as well as a joint search [11] exclude the possibility that the flux can be generated by a small number of bright sources. A first search employing a two-point correlation function with data from the ANTARES neutrino telescope [12] has neither found significant deviations from isotropy in the full-sky neutrino distribution nor shown evidence for correlations with catalogues of various astrophysical objects. Likewise a two-point correlation and multipole analysis of IceCube skymaps confirmed that the assumption of a small number of bright sources is excluded [13]. Therefore, a distribution of many faint point-like or unexpected extended sources constitutes a promising hypothesis at this stage.

ANTARES [14] is the largest operational neutrino telescope in the Northern Hemisphere, located in the Mediterranean Sea $\left(42^{\circ} 48^{\prime} \mathrm{N}, 6^{\circ} 10^{\prime} \mathrm{E}\right)$ at a depth of $2475 \mathrm{~m}$. Due to its location, it mainly observes the Southern sky in the upgoing muon channel and provides an excellent view of the Galactic Centre. Despite its much smaller instrumented volume compared to IceCube, it has an effective area for muon neutrinos which is comparable to that of the IceCube HESE analysis for energies around $100 \mathrm{TeV}$ and even surpasses it for energies below about $60 \mathrm{TeV}$ [15].

This paper introduces three independent new methods and the results obtained with them. The first two algorithms enhance the data selection and reconstruction process, while the third is a novel analysis method, referred to as multiscale method in the following, that uses the arrival directions of upgoing muon neutrinos recorded with the ANTARES neutrino telescope in 6 years of data taking. 


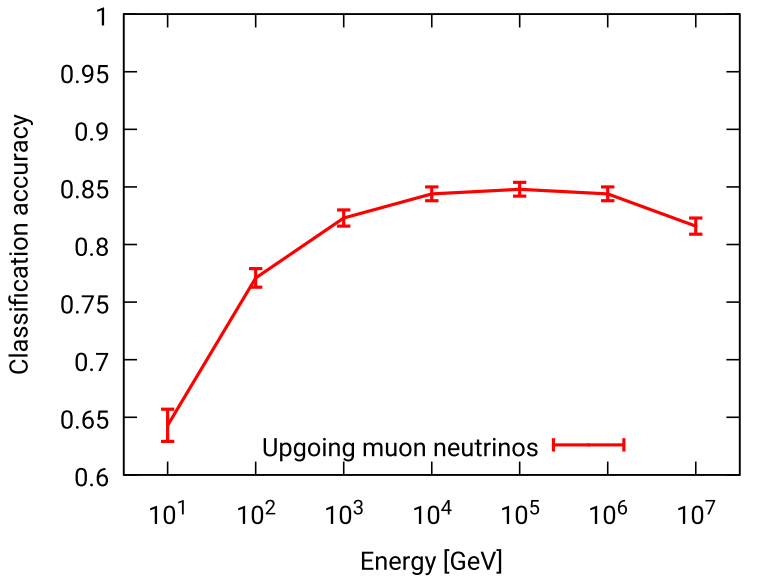

Fig. 1. The classification accuracy of the two step RDF classification for upgoing muon neutrinos versus neutrino energy for Monte Carlo simulations. The error bars indicate one standard deviation of statistical errors plus an estimate of the systematic error resulting from fluctuations in the training sample.

The goal of this analysis is to identify the most significant, spatially confined excess over the background of atmospheric neutrinos without relying on assumptions of any emission model of potential neutrino sources. The analysis method is sensitive to a clustering of events in an extended sky region, and its sensitivity increases with the size of the signal region. The multiscale method does not rely on any property derived from modelling or simulations, but computes all required estimates from the observed data. Compared to other searches for potential extended sources, it has the benefit of not being restricted to a given template. In contrast to other model-independent searches, like e.g. a two-point correlation analysis, it identifies the region of a dense clustering. The method is designed to yield a first indication of a candidate source region significantly deviating from the expected background distribution, but not to provide a detection with maximum significance or to interpret the nature of a candidate cluster.

\section{Data selection and reconstruction}

\subsection{Signal identification}

In order to distinguish events resulting from genuine neutrinos from the background of atmospheric muons that reach the telescope from above and generate about $10^{6}$ times more events, cuts are placed on the direction reconstruction to select those that are consistent with an "upgoing" particle entering the telescope from below.

In this analysis a multivariate classification technique called "Random Decision Forest" (RDF) ${ }^{1}$ [17] is used in addition to cuts on the reconstruction quality to allow for less strict cuts, increasing the available statistics. The RDF operates on variables describing the topology and timing pattern of the light observed within ANTARES [18]. The output of this algorithm for a recorded event is an assignment to a predefined class. In this application the classes are "upgoing" and "downgoing". The algorithm is trained on Monte Carlo simulations that incorporate the observed time-dependent data-taking conditions [19]. To improve the accuracy of the results, a two-step classification is used, where the first classification rejects only clearly downgoing signatures, while the second step is trained specifically to filter out those atmospheric muon events that generate patterns similar to the desired upgoing muon neutrinos. This technique reaches a rejection rate of $99.85 \% \pm 0.12 \%$ for

\footnotetext{
1 The used implementation is forked from an open source version of alglib [16].
}

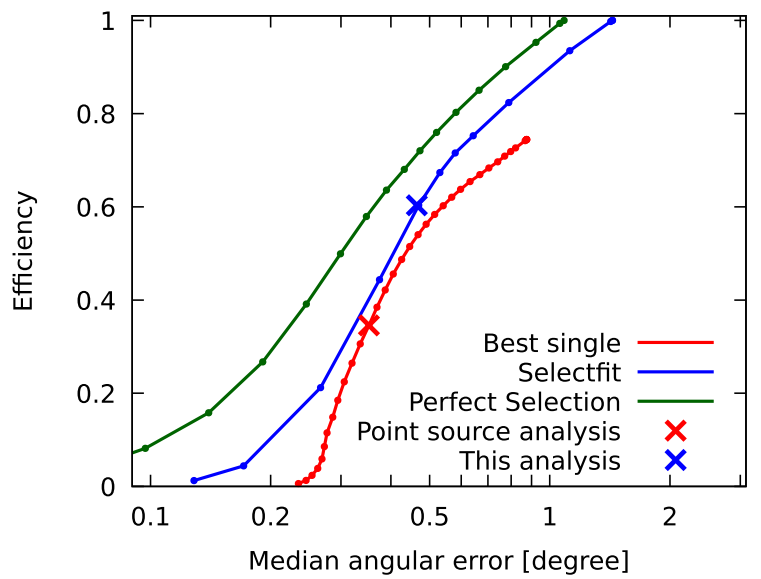

Fig. 2. Comparison of the efficiency versus the median angular error of direction reconstruction algorithms. Every point of these curves is obtained for a different cut on the quality variables. Shown are the performance of the on average most accurate individual direction reconstruction algorithm [20] in red for cuts on its standard quality parameter and the novel method "'Selectfit" with cuts on the estimated error class in blue. The crosses indicate the cuts used for the single algorithm in [23] and for Selectfit in this analysis. The green line shows the unreachable limit for the perfect combination of direction reconstruction algorithms. It is the performance if for every event the best available single reconstruction was chosen and the true angular uncertainty was used for the selection. All performances have been evaluated for a neutrino flux following an $E^{-2}$ energy spectrum. Efficiency is defined with respect to all triggered events where at least one direction reconstruction succeeded. (For interpretation of the references to colour in this figure legend, the reader is referred to the web version of this article.)

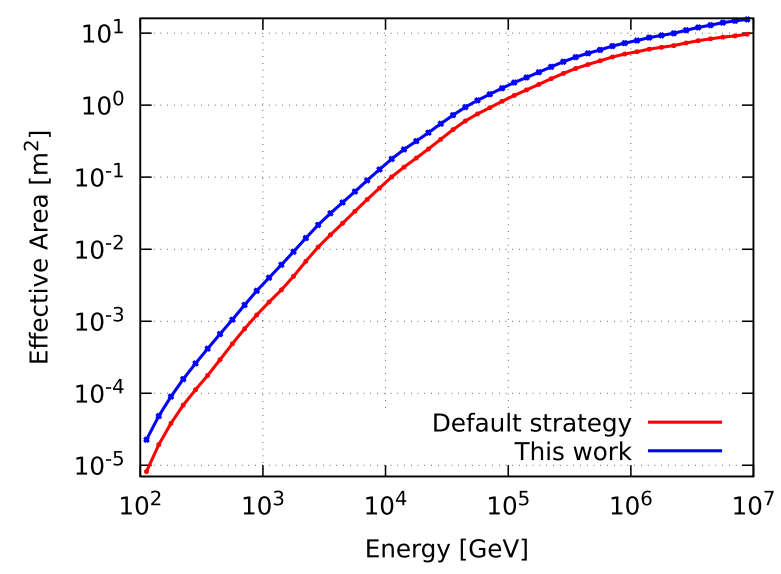

Fig. 3. Comparison of the effective area for the default event selection and reconstruction strategy for point sources [23] in red and this work in blue. The ratio is about 2.5 at $100 \mathrm{GeV}$ and decreases to about 1.5 for energies above $100 \mathrm{TeV}$. (For interpretation of the references to colour in this figure legend, the reader is referred to the web version of this article.)

downgoing atmospheric muons while preserving $81.7 \% \pm 1.3 \%$ of all charged current muon neutrino events (all numbers with respect to all triggered events, calculated for a spectrum following $E^{-2.0}$ ). Compared to a single stage RDF classification with a similar efficiency for upgoing neutrino events it reduces atmospheric muons by a factor of 20. Fig. 1 shows the efficiency for upgoing muon neutrinos as a function of the neutrino energy. The classification accuracy is defined as $\frac{\text { Number of correctly classified events }}{\text { Total number of events }}$.

\subsection{Direction reconstruction}

A novel method called "Selectfit" is used to reconstruct the direction of neutrino candidates. Instead of applying one reconstruction algorithm for all neutrino candidates, Selectfit combines the results of multiple direction reconstruction algorithms, aim- 


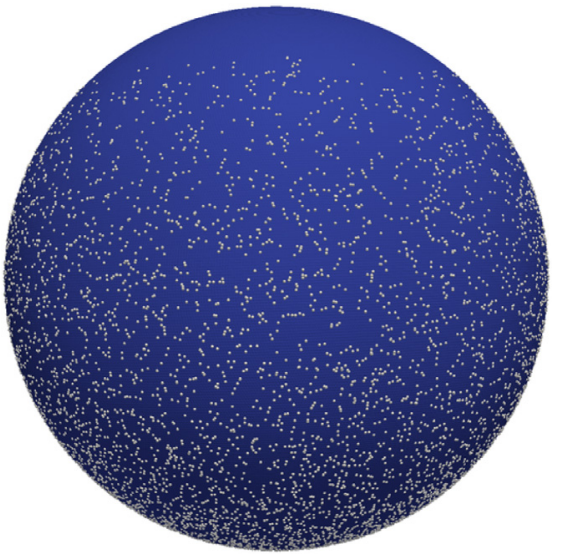

Fig. 4. A spherical grid in equatorial coordinates (in blue) with 12,000 randomly generated events and two point-like sources (in white). The grid points are rendered with a radius of about $0.5^{\circ}$, hence they overlap and form a closed sphere. Only the hemisphere of the three dimensional sphere facing the observer is visible in this near-side general perspective projection. View on the equator (declination of $0^{\circ}$ ). (For interpretation of the references to colour in this figure legend, the reader is referred to the web version of this article.)

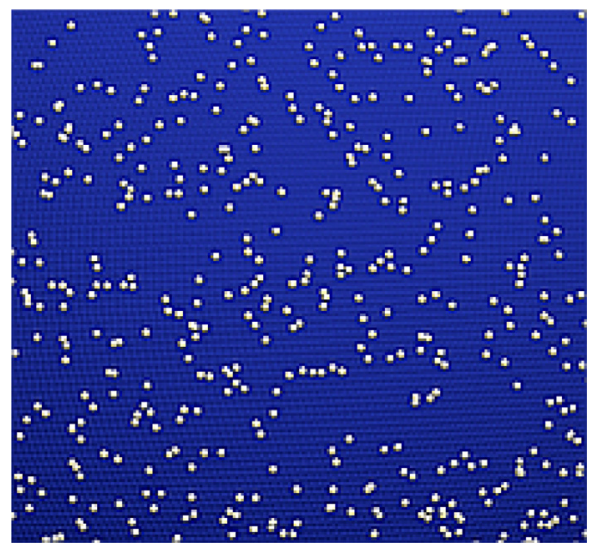

Fig. 5. A zoom to the centre of Fig. 4 where the grid points become visible.

ing to select the most precise result for each event. It combines four reconstruction algorithms previously used by ANTARES [2022 ]. While the reconstruction schemes of the algorithms that are combined are similar, each algorithm performs best for a different energy range or has different prerequisites for a successful reconstruction, allowing Selectfit to improve the overall result. The selection is again performed by a Random Decision Forest using the reconstruction results (zenith and azimuth angle) as well as all available quality-related output parameters of the considered reconstruction algorithms as input variables. It tries to identify the most accurate reconstruction result. The output class number hence determines which algorithm is used for an event.

In order to estimate the accuracy of the reconstruction for each event in a comparable way, Selectfit cannot just rely on the quality variable of the chosen algorithm. Instead it combines the results and quality parameters of all considered algorithms using a RDF. The output of this second RDF are classes corresponding to bins of angular error $\left(<0.1^{\circ},<0.2^{\circ},<0.4^{\circ},<0.8^{\circ}, \ldots\right)$.

As illustrated in Fig. 2, Selectfit either allows the angular error for a sample of neutrinos to be reduced or it can be used to increase the sample size for a given angular error. It increases the available statistics by at least $11 \%$ for any given accuracy. The benefit for small angular errors is mainly due to the improved estimation of the angular error, whereas for less accurate reconstruc-

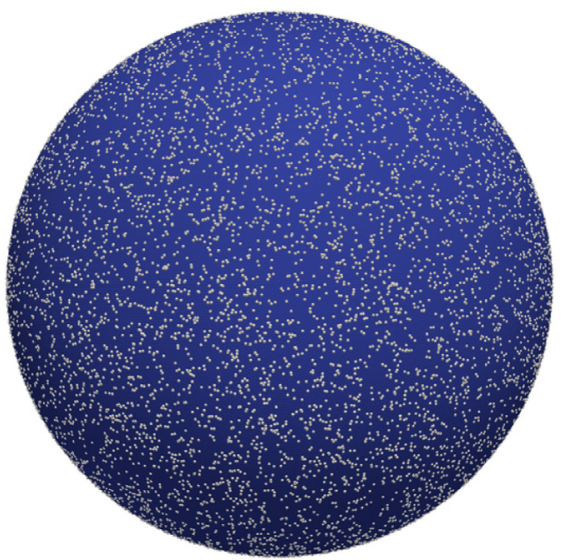

Fig. 6. The same setup as in Fig. 4, view from below on the south pole (declination of $-90^{\circ}$ ). This setup contains the artificially added events as shown more clearly in Fig. 7. All following spheres are oriented as in this Figure.

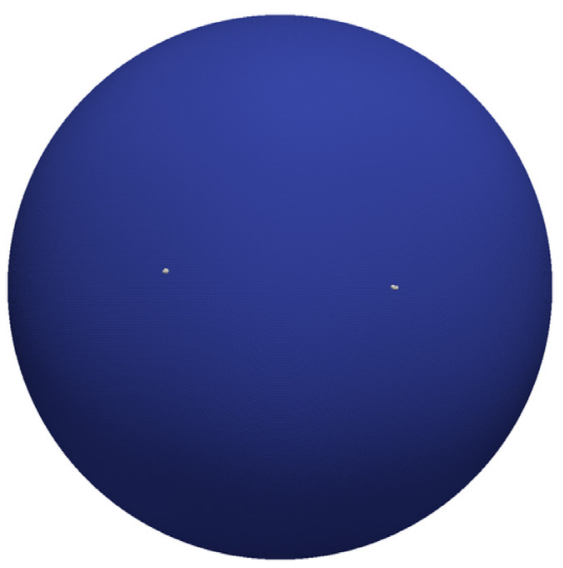

Fig. 7. The same setup and orientation as Fig. 6, but displayed without the random events. The remaining white points are the events of the two added point sources.

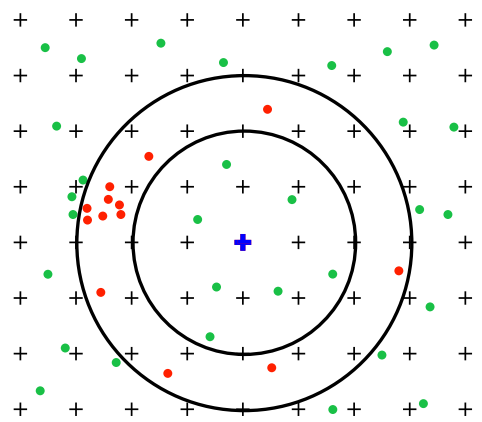

Fig. 8. Scheme of the neutrino counting. Crosses mark the grid points with a distance of $0.5^{\circ}$ between them. Green and red dots correspond to neutrinos. The blue cross is the grid point that is being evaluated. The search scale (here $1.0^{\circ}$ to $1.5^{\circ}$ ) is defined by the black circles. Neutrinos which are counted with the current search scale around the search point are shown in red. The results of the evaluation of this scale at the blue grid point is 13 . (For interpretation of the references to colour in this figure legend, the reader is referred to the web version of this article.)

tions, the main benefit results from the event-wise selection from the four reconstruction methods [18].

Since a search for extended sources does not necessarily require the same angular precision as a point source search, a looser cut on the uncertainty of the reconstruction is applied. For a flux of muon neutrinos with an energy spectrum of $E^{-2}$ these cuts result in a median angular uncertainty of $0.46^{\circ}$. The cut for this analysis was chosen to yield a high statistics sample of muon neutrino 

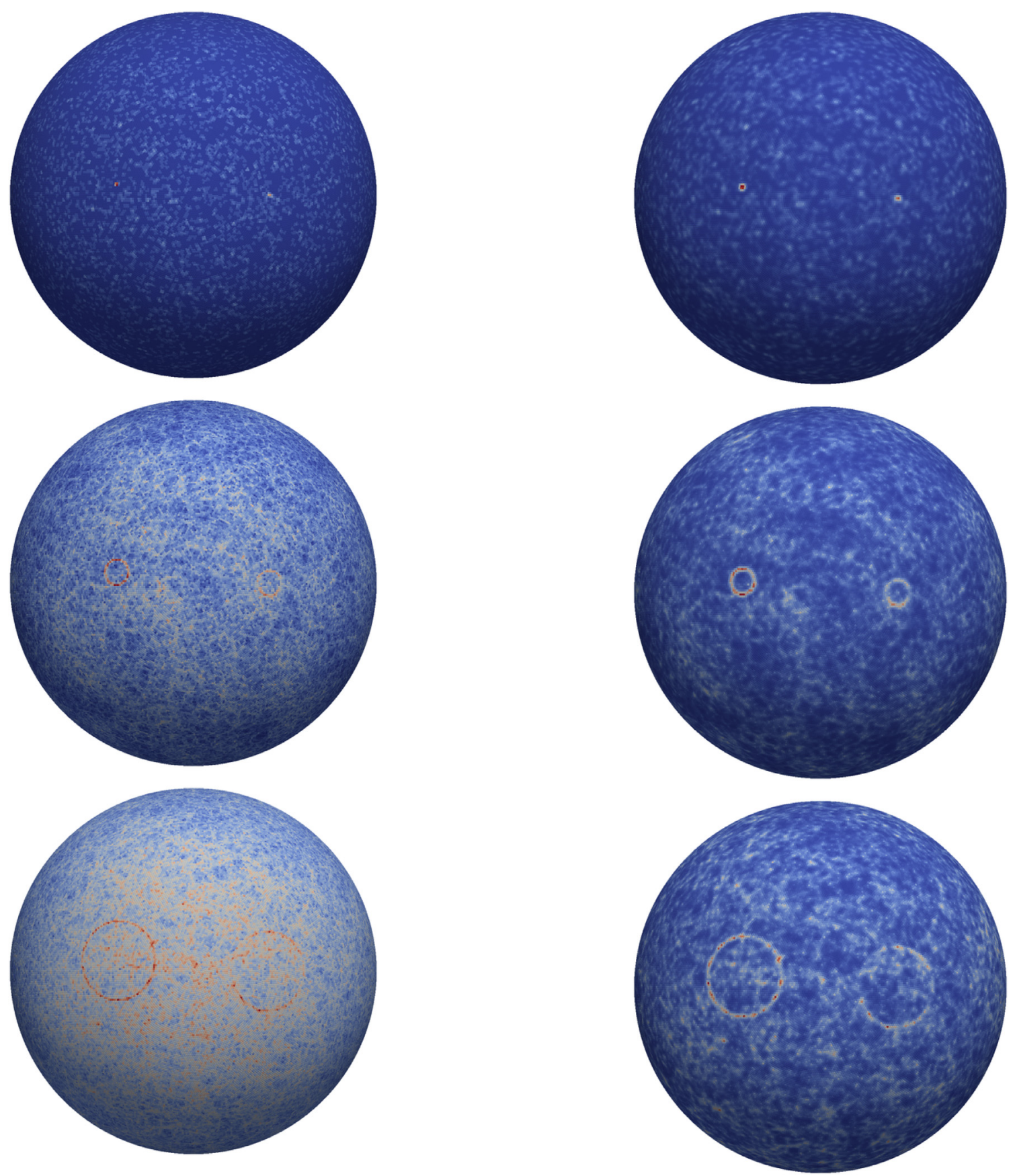

Fig. 9. The spherical search grid after counting the events presented in Fig. 6 in rings between $0^{\circ}$ and $0.5^{\circ}$ (top), between $3^{\circ}$ and $3.5^{\circ}$ (middle and between $10^{\circ}$ and $10.5^{\circ}$ (bottom) around each grid point. The colour code is readjusted from top to bottom to the range of values present on each sphere.

candidates while reconstructing them accurately enough to match the $0.5^{\circ}$ grid spacing used in the multiscale method discussed in the following section. In Fig. 3 the effective area reached with this method is compared to the effective area obtained with the default reconstruction scheme as used for point-source searches [23]. The looser event selection in this analysis also results in a higher background from misreconstructed atmospheric muons in the final sample of neutrino candidates (29.7\% instead of $10 \%$ in [9]).

\section{Model-independent multiscale source search}

The model-independent multiscale source search aims to identify the region of arbitrary position, size, shape and and distribu-

Fig. 10. Colour coded $R$ values as defined in Section 3.3 derived from the event counts shown in Fig. 9 for the scales $0^{\circ}$ to $0.5^{\circ}$ (top), $3^{\circ}$ to $3.5^{\circ}$ (middle), and $10^{\circ}$ to $10.5^{\circ}$ (bottom).

tion of neutrino events within the region with the most significant excess of neutrino events in the sky with respect to the background expectation.

The method itself $^{2}$ is independent from the ANTARES neutrino telescope or the fact that the analysed data are neutrino candidates. The only assumption made in this method is that the density of background events at any declination is approximately independent of the right ascension. Unlike many techniques known from image processing, it is designed to operate also on the sparse

\footnotetext{
2 The source code of a slightly altered version of this method can be found at https://github.com/sgeisselsoeder/multiscale and the method can easily be tested using https://hub.docker.com/r/km3net/multiscale.
} 

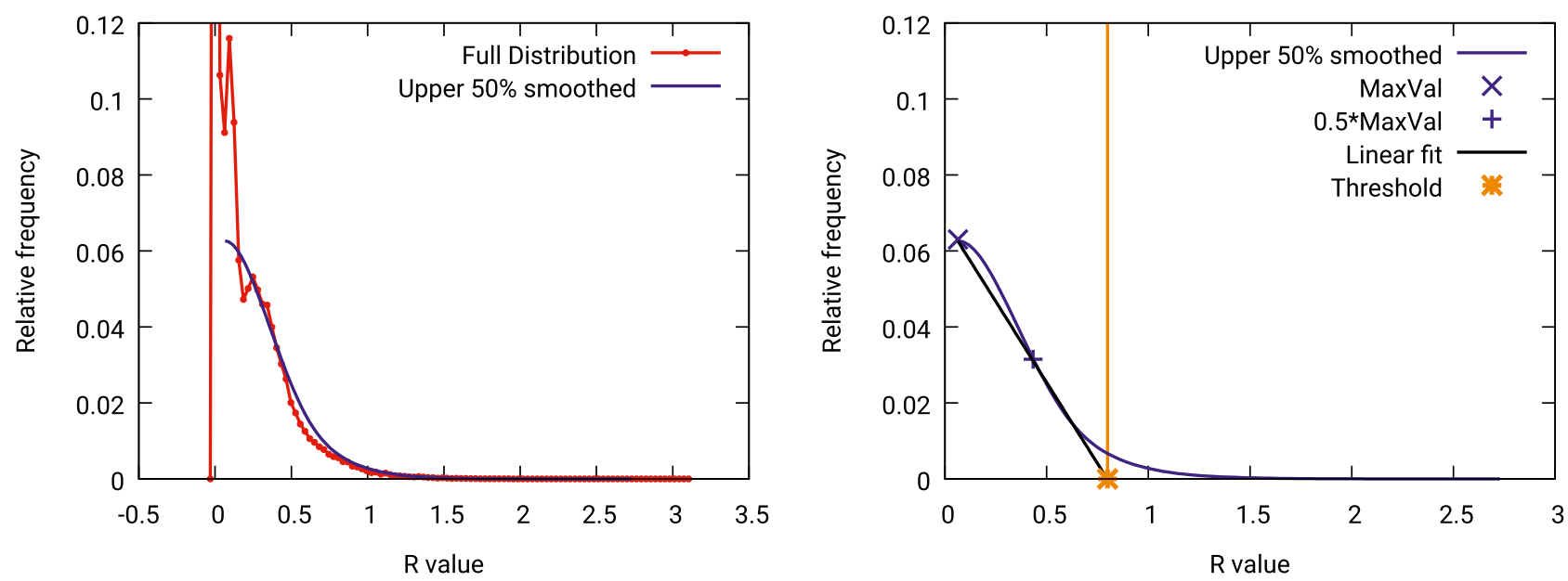

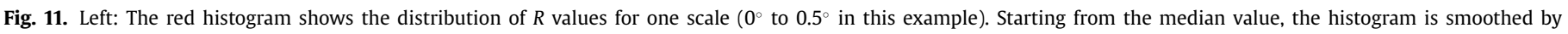

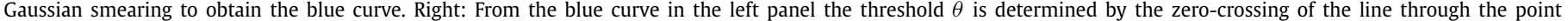

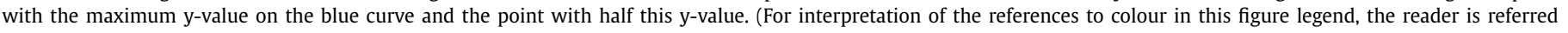
to the web version of this article.)

dataset of neutrinos. While many other fields of astronomy can operate on image-like measurements, these neutrino data would fill only very few grid points of a skymap. Hence many methods successfully used in other experiments are not applicable here.

The search method consists of eight subsequent steps applied to the measured neutrino sky map, described in subsections 3.13.8. These steps have been defined such that they are independent from assumptions on signal topology or spectrum. They implement measures to suppress background and reduce fluctuations, to identify regions that stick out from the background, and to determine topology and significance of a possible signal. They are the result of an extensive investigation and optimisation of different methods and options, as described in [18]. Since the resulting algorithm is highly complex and not targeted at a specific signal configuration, however, it is impossible to explore whether it is optimal in the full phase space of all possible analysis methods.

Compared to the standard ANTARES point-source searches [23], the multiscale method has been estimated to require a typically $20-100 \%$ higher neutrino flux in order to make an equally significant discovery for a point source, depending on the assumed source spectrum. However, these previous searches all target specific hypotheses of neutrino production and are not designed to detect unexpected sources or distributions of sources.

\subsection{The search grid}

In the first step a spherical grid is defined that allows for describing the sky map in terms of sets of integers assigned to the grid points (see subsequent steps). The distance of adjacent grid points is chosen to be $0.5^{\circ}$, commensurate with the angular resolution of reconstructed neutrino-induced muon tracks in ANTARES (see Fig. 2). The grid consists of 165016 grid points. Figs. 4-6 show the spherical grid, with grid points in blue and 12000 randomly generated neutrino events in white. This number of neutrinos is close to the expectation for the data analysis and they are distributed according to the visibility of ANTARES. In order to better illustrate the steps of the analysis method, two artificial point sources with 12 and 18 events, respectively, have been added at a declination of $-70^{\circ}$, see Figs. 6 and 7.

\subsection{Counting}

In this step, a set of integers is determined for each grid point by counting the neutrino events located in rings of different radii around that point; these integers will be analysed in the following using Poissonian statistics. The inner ring radius is referred to as the search scale, while the outer radius is $0.5^{\circ}$ larger. The search evaluates 180 scales, i.e. 180 different regions with increasing angular radius from $0^{\circ}$ up to $90^{\circ}$ in steps of $0.5^{\circ}$. This choice yields sensitivity to source regions up to an extension of twice the maximal search scale, i.e. $180^{\circ}$. The counting is performed for each grid point and for each search scale. The results are stored on an independent spherical grid for each search scale. A visualisation of the counting scheme is depicted in Fig. 8. For three scales the results for the example from Fig. 6 are shown in Fig. 9. Note that, unless stated otherwise, in these and all following similar figures, the colour code is readjusted between the different scales to match the full range of values present on each sphere.

\subsection{Poisson probabilities}

Poissonian probabilities $P(N, \lambda)$ are now used to infer a measure for the probability that the event numbers $N$ from the previous step exceed the background expectation. The expected mean number $\lambda$ for each grid point is estimated by pseudo-experiments using scrambled events. The scrambling is achieved by randomly shuffling the event times before converting from local to equatorial coordinates. This results in randomised distributions that preserve the characteristics of the actual data taking, for instance the distribution of the declinations or the efficiency of the data taking versus time. From the $P(N, \lambda)$, the parameter

$$
Q=\log _{10}\left(\frac{1}{P(x \geq N, \lambda)}\right)=-\log _{10}(P(x \geq N, \lambda))
$$

is calculated. Subsequently, a low-pass filter ${ }^{3}$ is applied to the $Q$ values on the spheres to reduce the influence of statistical fluctuations. These smoothed $Q$ values are called $R$ and serve as input for the next step. The search spheres after these computations are shown in Fig. 10.

\subsection{Segmentation $I$}

The next step aims to focus on potentially relevant information and to remove background fluctuations. In general, this task,

\footnotetext{
${ }^{3}$ Implemented as normalised box filter (averaging the values of eight neighbouring grid points and the grid point itself).
} 

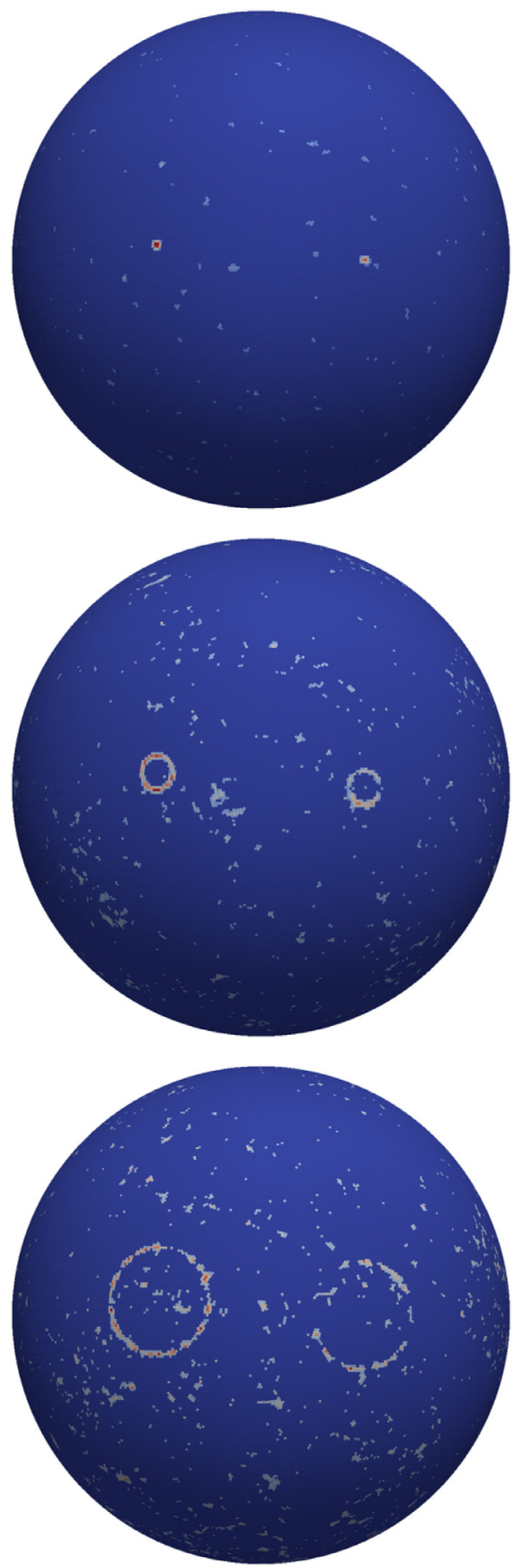

Fig. 12. $S$ maps after applying the segmentation step to the data shown in Fig. 10 according to Eq. (2) for the scales $0^{\circ}$ to $0.5^{\circ}$ (top), $3^{\circ}$ to $3.5^{\circ}$ (middle), and $10^{\circ}$ to $10.5^{\circ}$ (bottom).

well known in the field of computer vision, is called segmentation. Here it is performed using a simple threshold $\theta$. Values below the threshold, e.g. less pronounced fluctuations, are set to zero. The threshold $\theta$ is derived and applied for each search scale independently, based on the histogram of the $R$ values calculated for this scale. Fig. 11 illustrates the details how the threshold is obtained. While multiple other options to determine a threshold from the histogram would be possible, no method could be identified to be significantly better than the others.

The outcome of this step at a grid point $p$ is given by:

$S_{p}= \begin{cases}R_{p}, & R_{p} \geq \theta \\ 0, & R_{p}<\theta\end{cases}$
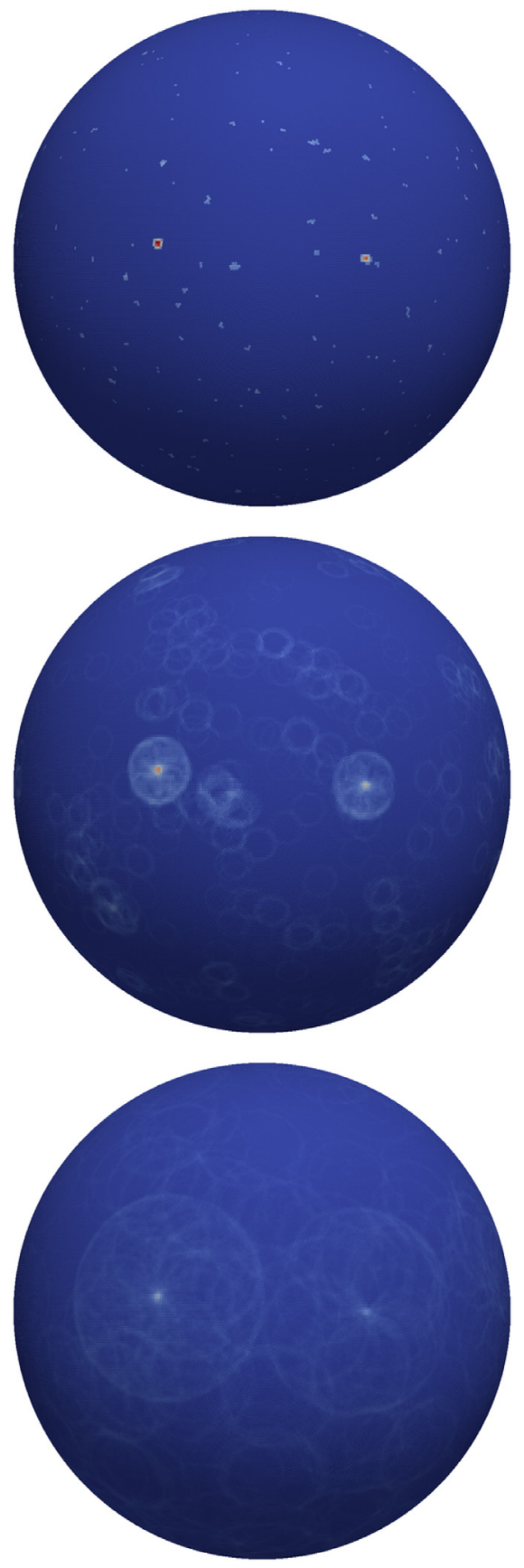

Fig. 13. Values of $T$ after the remapping step applied to the data shown in Fig. 10 according to Eq. (3), for the scales $0^{\circ}$ to $0.5^{\circ}$ (top) $3^{\circ}$ to $3.5^{\circ}$ (middle), and $10^{\circ}$ to $10.5^{\circ}$ (bottom). Note that the colour code is the same for all three pictures.

The resulting $S$ maps after the segmentations are shown in Fig. 12.

\subsection{Remapping}

The $S$ maps clearly show indications of the signal, but in a relocalised way - for example, point sources are mapped to circles with the radius corresponding to the search scale. The next step is to reconstruct the original location of the neutrinos that caused the remaining high values of $S$ on the spheres. Since the values for a given search scale $d$ originate from neutrinos that are between $d$ and $d+0.5^{\circ}$ away from the grid points where the values are stored, the following approach is used: let Set $t_{p}$ be the set of $N_{p}$ grid points with a distance between $d$ and $d+0.5^{\circ}$ around a grid 


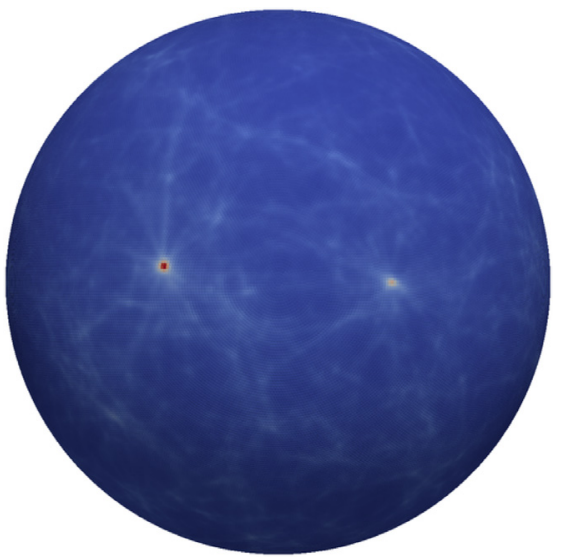

Fig. 14. Values of $U$, given by Eq. (4).

point $p$ and let $q$ be an element (a grid point) of this set. The value $T_{p}$ for grid point $p$ is calculated by averaging over the values $S_{q}$ of all grid points in $\operatorname{Set}_{p}$ :

$T_{p}=\sum_{q \in \operatorname{Set}_{p}} \frac{S_{q}}{N_{q}}$.

This step maps the information back to all potential origins, meaning all grid points where the neutrinos contributing to the value could have been located. Results of these computations are shown in Fig. 13. A higher density in the original neutrino distribution is now encoded in the overlapping pattern of the remapped circles as seen in the middle and bottom pictures of Fig. 13. Note that the halos around the original source positions are unavoidable artifacts of this method, which reduce its sensitivity for point-source searches but less so for extended sources.

\subsection{Summation}

So far, all calculations have been made independently for each search scale. In order to derive information on size and topology of potential signal regions without imposing a distance or size scale, the results for the different search scales need to be combined. Having evaluated multiple approaches to exploit the information available in the multitude of scales, a simple sum of the maps assigned to all scales of a given spherical grid was found to yield the most robust evaluation. Since the influence of random fluctuations is high for the smallest scale, it is not included in this sum. The input for the final steps is calculated as:

$U_{p}=\sum_{i=2}^{180} T_{p, i}$

where the index $i$ denotes the distance scale, given by $d=(i-1)$. $0.5^{\circ}$. This index has been omitted in the previous equations as all computations have been restricted to the same distance scale. The result of the summation according to Eq. (4), applied to the full data set and all scales, can be seen in Fig. 14 .

\subsection{Segmentation II}

A further segmentation is performed to isolate potential signal regions from background fluctuations. The same procedure which was used before for the histograms of $R$ values (see Section 3.4) is used here for the single histogram of $U$ values. In this step, however, different thresholds $\theta_{\beta}$ are used, obtained by scaling the difference between the minimum non-zero value found on the
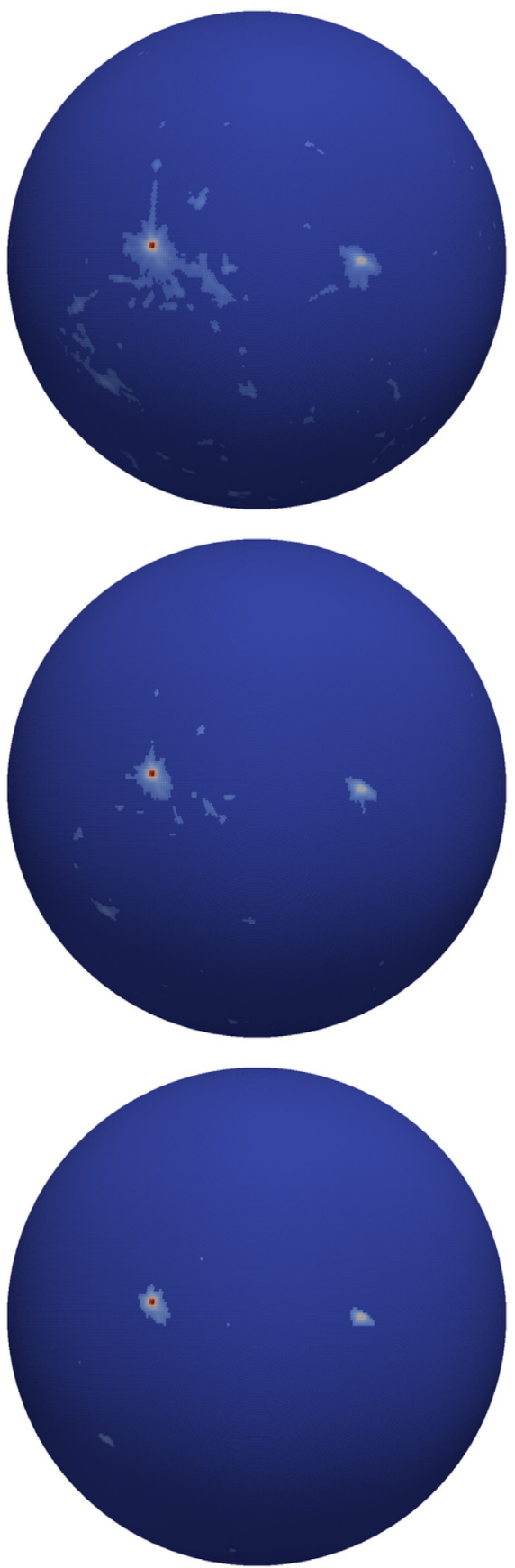

Fig. 15. $V$ values after the segmentation as given by Eq. (6) for $\beta=0.75$ (top), $\beta=$ 0.95 (middle), and $\beta=1.11$ (bottom).

sphere, $U_{\min }$, and $\theta$, the threshold computed as in the previous segmentations, by a factor $\beta \in \mathbb{R}$ :

$\theta_{\beta}=U_{\min }+\beta\left(\theta-U_{\min }\right)$.

The variable threshold $\theta_{\beta}$ serves to adjust the sensitivity of the segmentation step. The result of the segmentation using $\beta$ for each grid point $p$ is given by:

$V_{p}= \begin{cases}U_{p}, & U_{p} \geq \theta_{\beta} \\ 0, & U_{p}<\theta_{\beta}\end{cases}$

Lower values for $\beta$ result in lower thresholds $\theta_{\beta}$, allowing more extended structures to be found, while higher values only preserve the high peaks, favouring smaller morphologies. The results are fi- 


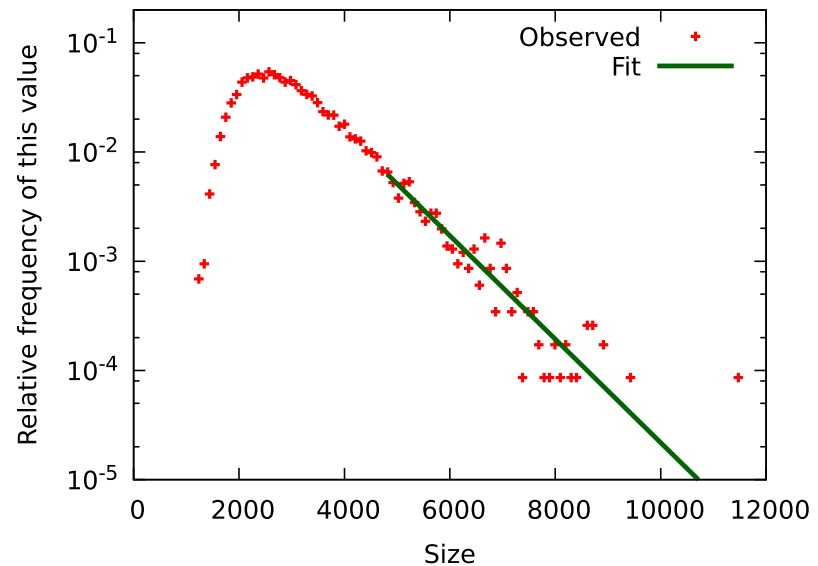

Fig. 16. The distribution of the sizes of clusters from pseudo-experiments and a fit to the tail of the distribution.

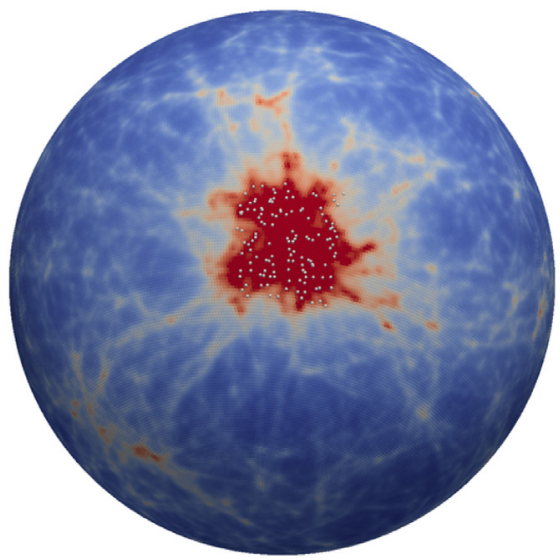

Fig. 17. An illustrative example for the behaviour of the method for an extended source, $20^{\circ}$ by $20^{\circ}$. The additional 120 source neutrinos are depicted in white. The 12,000 background neutrinos are not rendered. Colour indicates the $U$ values after the summation, corresponding to Fig. 14.

nally filtered using a two-dimensional median filter ${ }^{4}$ to suppress potential artefacts (e.g. single grid points). The effect of different thresholds for the segmentation can be seen in Fig. 15. Choosing the value of $\beta$ is the first step in this analysis where an explicit bias for any source property is included. Based on an evaluation of a variety of simulated sources, two values are used: $\beta=0.75$ and $\beta=1.11$. These values are chosen to be more sensitive to extended than to small (or point-like) structures. This choice is motivated by the objective to perform a search that complements the previous, location-independent ANTARES searches for point-like sources [23].

\subsection{Significance}

A possible signal of the analysis will manifest itself as a connected group of grid points, all with values above $\theta_{\beta}$; such an object is called a "cluster".

The final step is to distinguish potentially significant clusters from random accumulations. Since the exact size, shape, position and composition of a cluster is highly unlikely to be reproduced using pseudo-experiments, more generic metrics must be used to evaluate the significance of a cluster. Many metrics have been designed and tested on a multitude of simulated sources [18], each with different sensitivities to different sources. No single metric

\footnotetext{
4 The value at a grid point is replaced by the median value of the eight neighbouring grid points and itself.
}

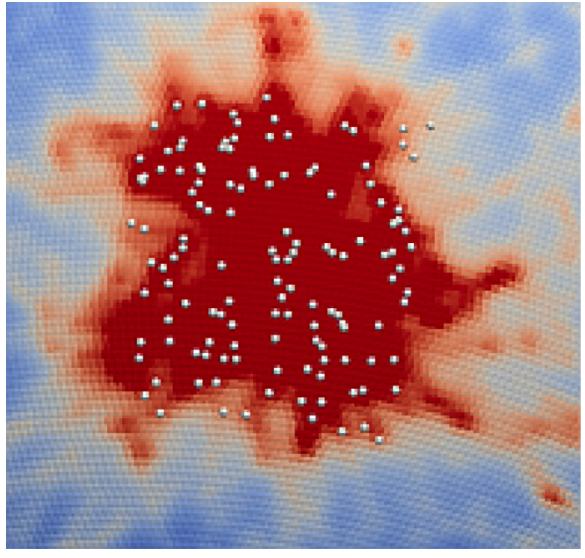

Fig. 18. A zoom to the source region of Fig. 17.
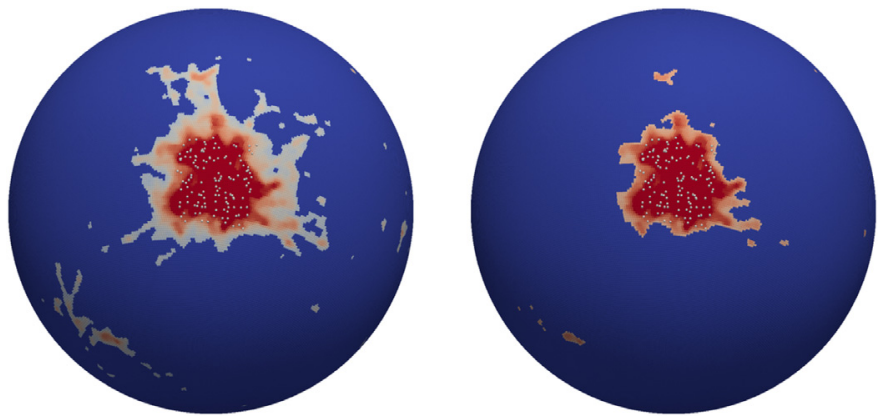

Fig. 19. The results for the setup in Fig. 17 after a segmentation using $\beta=0.75$ (left) and $\beta=1.11$ (right), respectively, corresponding to the $V$ values in the top panel of Fig. 15.

can be maximally sensitive to all potential sources. Motivated by the focus on extended sources, the metric "clustersize", given by the number $N_{c}$ of grid points in a cluster, has been chosen, performing best for extended source topologies. The quantity $N_{c}$ is mainly correlated wit the size of the signal region. Some information on the density of neutrinos within a cluster is nevertheless retained, because the average value of $U$ in a given cluster, and hence the cluster size, grows with the neutrino density.

The significance for a cluster is derived from pseudoexperiments with scrambled events as explained in Section 3.3. For each threshold $\theta_{\beta}$ the distribution obtained from the pseudoexperiments has to be treated independently, as e.g. the distribution of the sizes of clusters depends on the used threshold. A pre-trial p-value and hence a pre-trial significance is computed for each observed cluster using the corresponding distribution. The distribution of the values obtained for the metric "clustersize" (in grid points) for a threshold using $\beta=0.75$ is shown in Fig. 16. An exponential function $a \cdot e^{b x}$ with free parameters $a$ and $b$ is fitted to the tail of the distribution. This function is used instead of the histogram if the significance determination would be limited by the available statistics. For each pseudo-experiment, only the highest pre-trial significance of any cluster from any threshold is considered the result of this pseudo-experiment. The post-trial significance is then obtained by a comparison of the measured pre-trial significance of an observed cluster with the distribution obtained from pseudo-experiments.

\subsection{Application to extended sources and general considerations}

To illustrate the behaviour of the method for a simulated extended source, the configuration shown in Figs. 17 and 18 has been investigated. As shown in Fig. 19, the location and size of the sim- 

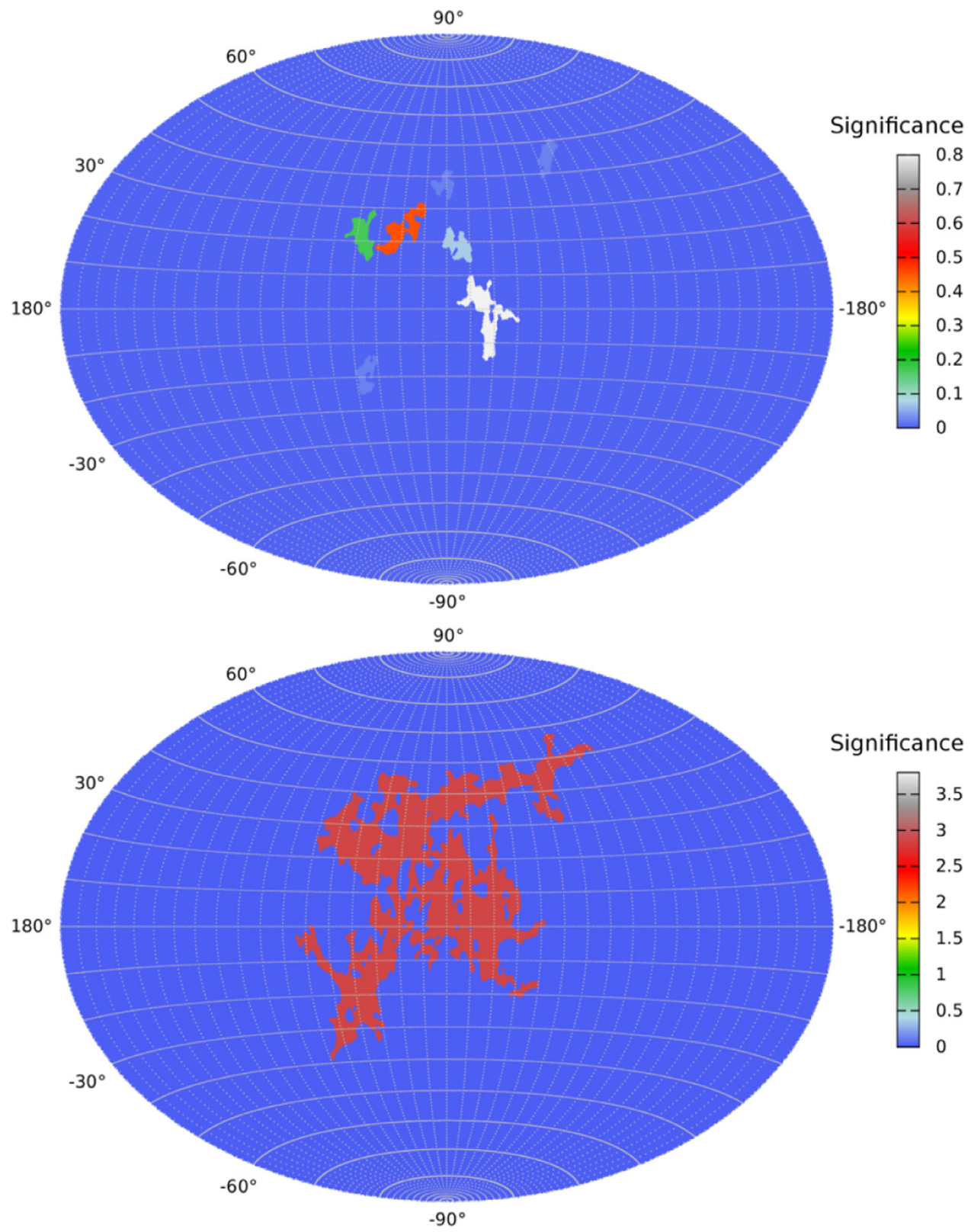

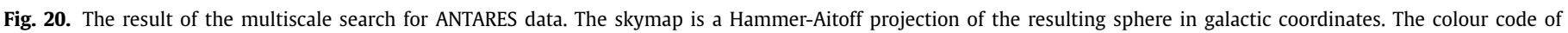
the clusters indicates the post-trial significance in units of $\sigma$. Top: using $\beta=1.11$ for segmentation. Bottom: Using $\beta=0.75$.

ulated signal are approximated reasonably well. For the result obtained with the lower $\beta$ value, however, additional filaments extend from the actual shape to regions where random accumulations of background neutrinos occurred.

The sensitivity of this method for deviations from a homogeneous spatial distribution is different compared to previously used algorithms. For instance, tests with the same simulated pseudoexperiments showed that the two-point correlation analysis [24] is more sensitive to scenarios where many faint sources with the same extension are distributed evenly throughout the sky. On the other hand, the multiscale search described here is more sensitive in scenarios that include a spatial clustering of these faint sources. It also benefits more from the presence of one or more dominant sources.

The additional information reported in [18] (pp. 95-98) verify an important aspect of the method: It only requires an increase in the number of excess events by a factor of six for an increase of the signal region from (2 degrees $)^{2}$ to $(20 \text { degrees })^{2}$. Since a factor of ten would be expected for a constant signal-to-noise ratio, it can be concluded that the multiscale search is most sensitive to largescale clusterings and thus has a unique sensitivity characteristic, clearly different from most other search methods.

More examples of sources, also for asymmetric shapes, and more details on the quantitative effect of neighbouring sources can be found in [18].

While the true nature of the sources of high-energy cosmic neutrinos is still unknown, this algorithm offers a high sensitivity for a wide range of possible scenarios, including ones in which many faint sources dominate the observed diffuse flux [25].

\section{Results}

This analysis was performed following a data blinding concept. That means that the development of all methods presented in Sections 2 and 3 and the optimisation of all cuts had been com- 

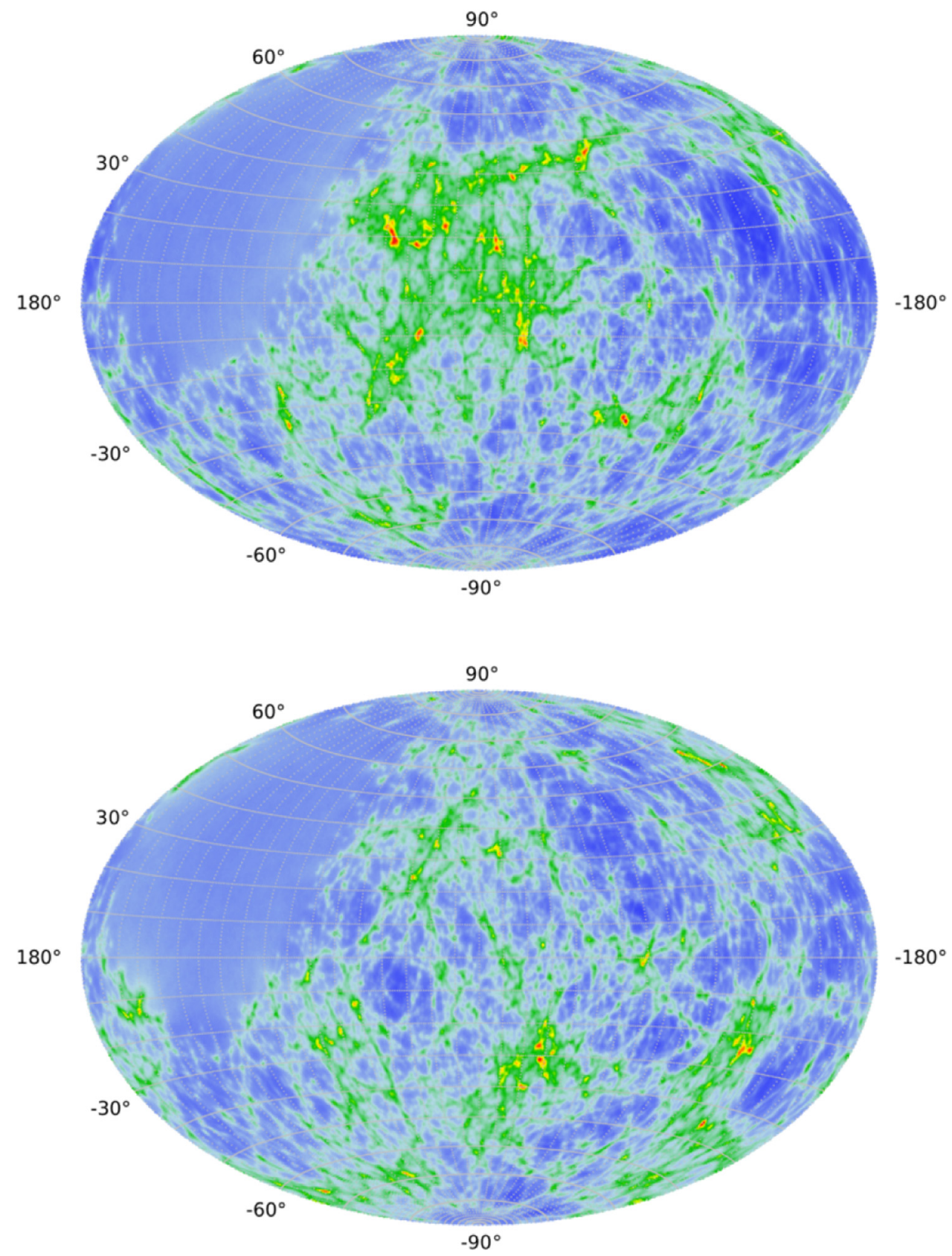

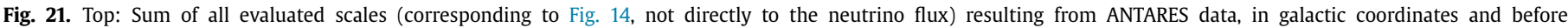

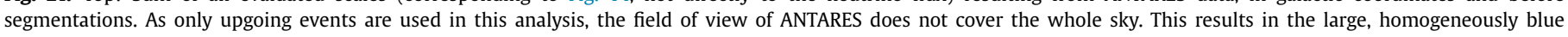

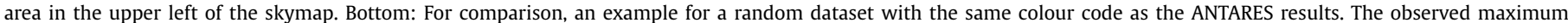

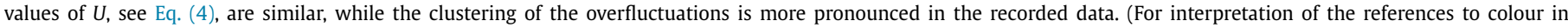
this figure legend, the reader is referred to the web version of this article.)

pletely finished before the recorded data sample was processed. The small data sample that had been used to verify the methods was excluded from the final sample.

\subsection{ANTARES}

Using the methods described in Section 2.1 and 2.2, the evaluation of the ANTARES data from 2007 to 2012 results in 13,283 candidates for charged current muon neutrino events, with an expected number of background neutrinos of $13078 \pm 362$ (statistical error) from interactions of cosmic rays in the atmosphere. This corresponds to a background expectation of 0.634 neutrino candidates/degree ${ }^{2}$. The multiscale search method described in Section 3 yields the result shown in Fig. 20. Using the higher segmentation threshold $\beta=1.11$, no cluster with a significance above $0.8 \sigma$ has been found. With $\beta=0.75$ a very large structure is found. After accounting for all known systematic effects, the large structure, called "the cluster" from here on, has a post-trial significance of $2.5 \sigma$. The investigation of the systematic effects can be found in [18]. The size of the cluster is 13,312 connected grid points, equivalent to about 3328 degree $^{2}$ or $8 \%$ of the sky.

The region contains about 200 events in addition to the about 2100 events expected from background. This corresponds to an excess of $0.06 \pm 0.015$ neutrino candidates/degree ${ }^{2}$. These numbers 

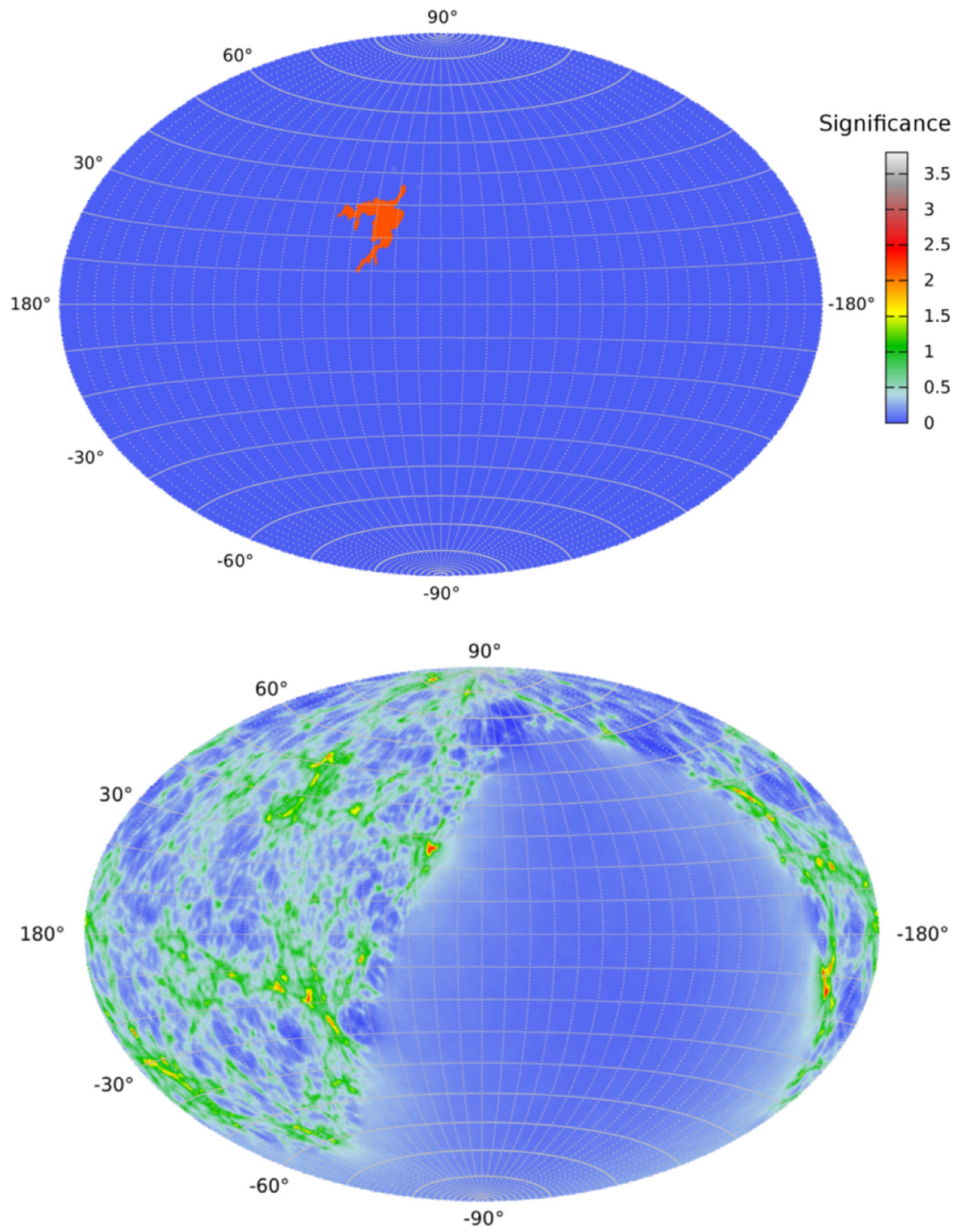

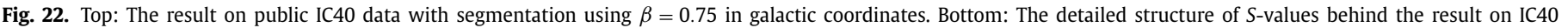
data before the segmentation.

are derived assuming no subclustering within the cluster. Any subclustering (e.g. by embedded smaller clusters) would result in the same significance for a smaller average neutrino excess density. This localized event excess would not have been noticed by the two-point correlation analysis [24] nor the point source analysis and does not violate the existing bounds and observations from ANTARES and Icecube on the astrophysical diffuse flux.

Even if not significant on its own, this structure constitutes an interesting feature in the data which is worth further studies. It can be noted that the cluster contains the Galactic Centre, which is located in the centre of the presented skymaps in galactic coordinates. More details on the inner structure of these clusters can be seen in Fig. 21, which shows the result of the summation of all scales before the segmentations. It should be noted that with the limited available statistics, random fluctuations do influence the results.

\subsection{IceCube IC40}

In order to perform an independent cross check of the result obtained using ANTARES data, the publicly available IC40 dataset [26] published by the IceCube Collaboration has also been analysed. This analysis searches specifically for an excess in the area of the large cluster found in ANTARES data. To achieve this, it only considers clusters found in the IC40 data that overlap with the ANTARES cluster by at least $51 \%$ of their area. The value of $51 \%$ is chosen here because this means that they are more related to the cluster from ANTARES than to other regions. On the other hand, requiring an overlap of close to $100 \%$ would exclude e.g. clusters that extend beyond the shape of the ANTARES cluster.

Since the requirement for overlap implicitly confines the size of the cluster, the clustersize in grid points, $N_{c}$, is no reasonable metric to assess the significance of a cluster in this evaluation. There- 
fore the metric that gave the second best results in the investigations introduced in Section 3.8 has been used. It is the mean value of the $\sqrt{N_{c}}$ grid points ${ }^{5}$ with the highest values within a cluster.

The result obtained by this adapted search on the IC40 dataset is shown in Fig. 22. A cluster is found within the expected shape with a post-trial significance of $2.1 \sigma$. The position of the found cluster overlaps with the search template by $78 \%$ of its size.

The observation that not all features in the skymaps in Figs. 21 and 22 match exactly is to be expected, as ANTARES has its highest sensitivity at lower energies compared to IceCube. Moreover, random fluctuations of atmospheric neutrinos do influence the results.

\section{Conclusions}

This paper presents three original and unpublished tools that have been used for a search for cosmic neutrino sources of arbitrary location and morphology by detecting the most pronounced deviation from the background-only expectation. The study is fully data-driven, without relying on any model for neutrino emission and input derived from simulations.

The first tool (see Section 2.1) is a multivariate classification technique used to increasing the available statistics of ANTARES data. The second (see Section 2.2) enhances the directional reconstruction of charged-current muon neutrino candidates. The third tool is a model-independent multiscale method (described in Section 3) that represents the core of this study.

Due to the fact that the method is independent of neutrino emission models or assumptions on the topology of emission regions, this analysis is not intended to discover potential sources with the highest achievable significance, nor to analyse the properties of a candidate source region. Instead, it aims at indicating the clustering in the data least expected from the background-only hypothesis and thus to initiate more specific investigations.

Applied to ANTARES data recorded between 2007 and 2012, this analysis found a large structure with a post-trial significance of $2.5 \sigma$. This result is consistent with a random fluctuation of the background of atmospheric neutrinos. Using this method to analyse public data from IceCube resulted in an excess located within the overlap between the cluster from the ANTARES data and the field of view of IceCube. This observation has a significance of $2.1 \sigma$.

Even though a general, model-independent analysis cannot be as sensitive as a dedicated search due to the high trial factors, this method provides a good way to become aware of the most prominent and even unforeseen structures in data and can be regarded as a trigger for more specific investigations. Despite the high trialfactor, it can outperform standard point-source and two-point correlation analyses in a scenario of unknown extended structures.

\section{Acknowledgments}

The authors acknowledge the financial support of the funding agencies: Centre National de la Recherche Scientifique (CNRS), Commissariat à l'énergie atomique et aux énergies alternatives (CEA), Commission Européenne (FEDER fund and Marie Curie Program), Institut Universitaire de France (IUF), IdEx program and UnivEarthS Labex program at Sorbonne Paris Cité (ANR-10-LABX0023 and ANR-11-IDEX-0005-02), Labex OCEVU (ANR-11-LABX0060) and the $A^{*}$ MIDEX project (ANR-11-IDEX-0001-02), Région Île-de-France (DIM-ACAV), Région Alsace (contrat CPER), Région Provence-Alpes-Côte d'Azur, Département du Var and Ville de La Seyne-sur-Mer, France; Bundesministerium für Bildung und
Forschung (BMBF), Germany; Istituto Nazionale di Fisica Nucleare (INFN), Italy; Stichting voor Fundamenteel Onderzoek der Materie (FOM), Nederlandse organisatie voor Wetenschappelijk Onderzoek (NWO), the Netherlands; Council of the President of the Russian Federation for young scientists and leading scientific schools supporting grants, Russia; National Authority for Scientific Research (ANCS), Romania; Ministerio de Economía y Competitividad (MINECO): Plan Estatal de Investigación (refs. FPA201565150-C3-1-P, -2-P and -3-P, (MINECO/FEDER)), Severo Ochoa Centre of Excellence and MultiDark Consolider (MINECO), and Prometeo and Grisolía programs (Generalitat Valenciana), Spain; Ministry of Higher Education, Scientific Research and Professional Training, Morocco. We also acknowledge the technical support of Ifremer, AIM and Foselev Marine for the sea operation and the CC-IN2P3 for the computing facilities.

\section{References}

[1] M.G. Aartsen, et al., First observation of PeV-energy neutrinos with IceCube, Phys. Rev. Lett. 111 (2013) 021103

[2] M.G. Aartsen, et al., Evidence for high-energy extraterrestrial neutrinos at the IceCube detector, Science 342 (2013) 1242856

[3] M.G. Aartsen, et al., Atmospheric and astrophysical neutrinos above $1 \mathrm{TeV}$ interacting in IceCube, Phys. Rev. D91 (2) (2015) 022001.

[4] M.G. Aartsen, et al., A combined maximum-likelihood analysis of the high-energy astrophysical neutrino flux measured with IceCube, Astrophys. J. 809 (1) (2015) 98.

[5] M.G. Aartsen, et al., Observation and characterization of a cosmic muon neutrino flux from the Northern Hemisphere using six years of IceCube data, Astrophys. J. 833 (1) (2016) 3.

[6] M.G. Aartsen, et al., Observation of high-energy astrophysical neutrinos in three years of IceCube data, Phys. Rev. Lett. 113 (2014) 101101.

[7] A. Neronov, D.V. Semikoz, Evidence the Galactic contribution to the IceCube astrophysical neutrino flux, Astropart. Phys. 75 (2016) 60-63.

[8] A. Neronov, D.V. Semikoz, Galactic and extragalactic contributions to the astrophysical muon neutrino signal, Phys. Rev. D 93 (2016) 123002.

[9] S. Adrián-Martínez, et al., Searches for Point-like and extended neutrino sources close to the Galactic Centre using the ANTARES neutrino Telescope, Astrophys. J. 786 (2014) L5.

[10] M.G. Aartsen, et al., All-sky search for time-integrated neutrino emission from astrophysical sources with 7 years of IceCube data, Astrophys. J. 835 (2) (2017) 151.

[11] S. Adrián-Martínez, et al., First combined search for neutrino point-sources in the Southern Hemisphere with the ANTARES and IceCube neutrino telescopes, Astrophys. J. 823 (2016) 65.

[12] S. Adrián-Martínez, et al., Searches for clustering in the time integrated skymap of the ANTARES neutrino telescope, JCAP 1405 (2014) 001.

[13] M.G. Aartsen, et al., Searches for small-scale anisotropies from neutrino point sources with three years of IceCube data, Astropart. Phys. 66 (2015) 39-52.

[14] M. Ageron, et al., ANTARES: The first undersea neutrino telescope, Nucl. Inst. and Meth. A 656(1) (2011) 11-38.

[15] S. Adrián-Martínez, et al., ANTARES constrains a blazar origin of two IceCube PeV neutrino events, A\&A 576 (2015) L8.

[16] V. Bystritsky, S. Bochkanov, (The ALGLIB Project) ALGLIB, 2011, (http://www. alglib.net). 2011-11.

[17] T.K. Ho, The random subspace method for constructing decision forests, IEEE Trans. Pattern Anal. Mach.Intell. 20 (1998) 832-844.

[18] S. Geißelsöder, Model-independent search for neutrino sources with the ANTARES neutrino telescope, Friedrich-Alexander-Universität ErlangenNürnberg, 2016 Ph.D. thesis. https://opus4.kobv.de/opus4-fau/files/6997/ dissertation geisselsoeder.pdf (in English, preface in German).

[19] L. Fusco, et al., The Run-by-Run Monte Carlo simulation for the ANTARES experiment, EPJ Web of Conf. 116 (2016) 02002.

[20] S. Adrián-Martínez, et al., First search for point sources of high energy cosmic neutrinos with the ANTARES neutrino telescope, Astrophys. J. Lett. L14 (2011) 743.

[21] J. Aguilar, et al., A fast algorithm for muon track reconstruction and its application to the ANTARES neutrino Telescope, Astropart. Phys. 34 (2011) 652-662.

[22] E.L. Visser, Neutrinos from the Milky Way, Universiteit Leiden, 2015 Ph.D. thesis. http://www.nikhef.nl/pub/services/biblio/theses_pdf/thesis_EL_Visser.pdf.

[23] S. Adrián-Martínez, et al., Search for Cosmic neutrino point sources with four year data of the ANTARES telescope, Astrophys. J. 760 (2012) 53.

[24] S. Adrián-Martínez, et al., Searches for clustering in the time integrated skymap of the ANTARES neutrino telescope, JCAP 05 (2014) 001.

[25] M.G. Aartsen, et al., Astrophysical neutrinos and cosmic rays observed by IceCube, Adv. Space Res. 62 (2018) 2902

[26] R. Abbasi, et al., A search for a diffuse flux of astrophysical muon neutrinos with the IceCube 40-string detector, Phys. Rev. D 84 (2011) 082001. http:/ icecube.wisc.edu/science/data/ic40.

\footnotetext{
${ }^{5}$ Rounded to the nearest integer number.
} 